\title{
Brain Abscess in a Patient with Rendu-Osler-Weber Syndrome: Value of Proton Magnetic Resonance Spectroscopy
}

\author{
Alex Roberto Ramírez Mejía, Miguel Yus Fuertes, and Manuela Jorquera Moya
}

The diagnosis of intracranial focal lesions based on imaging studies is a challenge. In brain abscess, proton magnetic resonance spectroscopy (1H-MRS) is useful to define etiology, differentiating anaerobic from aerobic pathogens and inclusive sterile abscess based on the behavior of certain metabolites. We report a patient with Rendu-Osler-Weber syndrome, pulmonary arteriovenous fistulas, and brain abscesses characterized by brain magnetic resonance imaging (MRI), with a spectroscopy pattern compatible with anaerobic infection.

Keywords: brain abscess, magnetic resonance spectroscopy, Rendu-Osler-Weber syndrome

\section{Introduction}

The differentiation between brain abscesses and other entities with ring-type contrast enhancement is difficult based only on image, because abscesses simulate other injuries as primary tumors or metastases. In contrast to magnetic resonance imaging (MRI) and computed tomography $(\mathrm{CT})$ that provide structural data, proton magnetic resonance spectroscopy (1H-MRS) gives completely different information related to neuronal integrity, cell proliferation or degradation, energy, metabolism, and necrotic transformation of brain or tumor tissues. 1H-MRS allows the study of metabolites normally absent in the normal brain, gives information on the nature of the abscess, and thus increases the specificity of conventional MRI studies. ${ }^{1)}$ $1 \mathrm{H}-\mathrm{MRS}$ can provide information about the cause of the abscess and their response to treatment, and since anaerobic bacteria typically have a growth pattern in culture media slower than aerobic bacteria, $1 \mathrm{H}-\mathrm{MRS}$ can be useful in choosing an appropriate antibiotic therapy. ${ }^{2)}$

\section{Case Report}

A 67-year-old-woman with known rheumatoid arthritis, anemia, and epistaxis was referred to the emergency department with a history of 10 days of drowsiness, clumsiness, dysthermia, transient loss of vision, and decreased strength in right limbs. Examination demonstrated right hemiparesis. She was referred to our institution with a brain CT that showed two rim enhancing lesions in left parietal and right occipital lobes with surrounding edema, suspecting brain metastases.

Department of Diagnostic Radiology, Hospital Clínico San Carlos, Madrid, Spain

Received: June 4, 2015; Accepted: September 20, 2015
Brain MRI was performed by using a 1.5-T MR imaging unit (GE Medical Systems, Milwaukee, Wisconsin, USA) with a quadrature $\mathrm{T} / \mathrm{R}$ head coil. Routine transverse and sagittal fast spin-echo (FSE) $\mathrm{T}_{1}$-weighted image (WI), transverse and coronal FSE $\mathrm{T}_{2} \mathrm{WI}$, transverse fluid attenuation inversion recovery (FLAIR) and diffusion weighted imaging (DWI), and post contrast transverse $\mathrm{T}_{1} \mathrm{WIs}$ three-dimensional fast spoiled gradient (3D-FSPGR) images were acquired after intravenous administration of gadobutrol (Gadovist; Bayer Pharma AG, Berlin, Germany) at a dose of $0.1 \mathrm{mmol} / \mathrm{kg}$ of body weight. The images were obtained with an acquisition matrix of $256 \times$ $256(128 \times 128$ on the DWI $)$ and a field of view of $24 \times 24 \mathrm{~cm}$.

Single-voxel volume-selective water suppressed 1H-MRS was performed by using PROBE-PRESS sequence with repetition time (TR) $1,500 \mathrm{~ms}$ and echo time (TE) $35 \mathrm{~ms}$, $144 \mathrm{~ms}$, and $288 \mathrm{~ms}$, and a voxel size of 8-12 ml (mean: $10 \mathrm{ml}$ ) in the center of the lesion. We used FLAIR images to locate the voxels for the 1H-MRS (Figs. 1, 2).
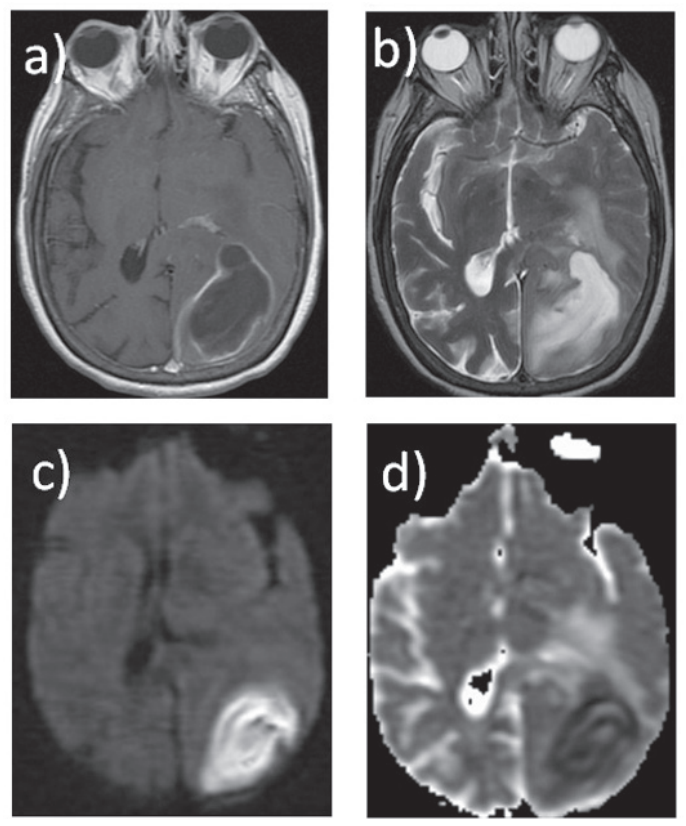

Fig. 1 a: Contrast-enhanced $\mathrm{T}_{1}$-weighted image (WI) shows a welldefined rim enhancing intra-axial mass in left parietal and occipital lobes with low intensity center, b: $\mathrm{T}_{2} \mathrm{WI}$ of the same lesion demonstrate vasogenic edema and intermediate to slightly low signal thin rim capsule, c: diffusion weighted imaging (DWI) sequence shows high signal intensity. d: Apparent diffusion coefficient (ADC) map of the lesion demonstrate restricted diffusion. 


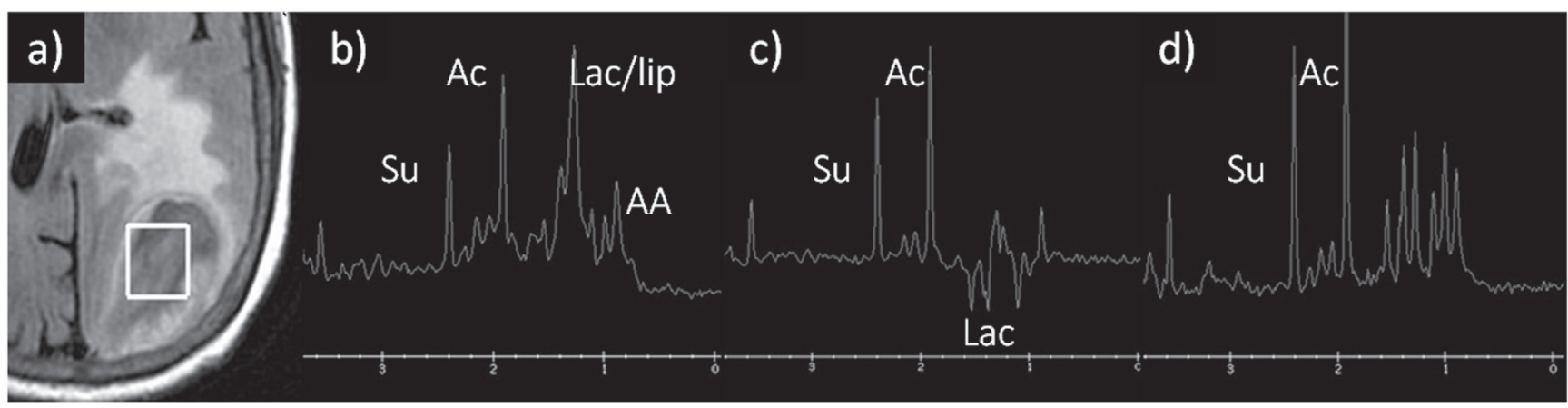

Fig. 2 a: Transverse FLAIR image shows the location of the rectangular voxel placed for 1H-MRS in the occipital lobe. b-d: Single-voxel 1H-MRS with echo times of (b) $35 \mathrm{msec}$ (c) $144 \mathrm{msec}$ and (d) $288 \mathrm{msec}$. The 1H-MRS spectrum shows absence of N-acetylaspartate (2.02 ppm), choline (3.2 ppm), and creatine (3.0 ppm) peaks, and elevation of amino acids (AA) (0.9 ppm), lactate (Lac) (1.33 ppm), acetate (Ac) (1.9 ppm), and succinate (Su) (2.4 ppm) peaks. Inversion of lactate peak with an echo time of $144 \mathrm{msec}$ in (c). Lac/Lip: lactate/lipid. ppm: parts per million, FLAIR: fluid attenuation inversion recovery, 1H-MRS: proton magnetic resonance spectroscopy.

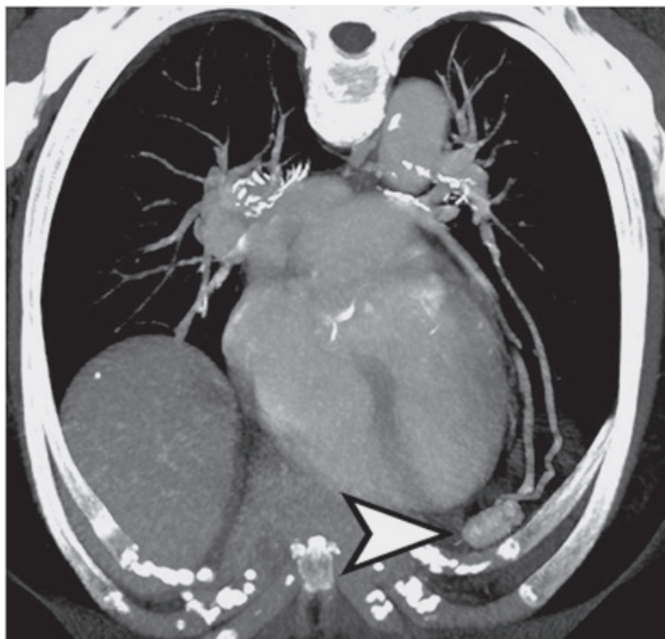

Fig. 3 Contrast-enhanced reformatted thoracic computed tomographic scan with mediastinal soft tissue window settings demonstrates a large pulmonary arteriovenous fistula (arrow) in the lingula.

A diagnosis of brain abscess was made, the 1 H-MRS findings were consistent with the presence of anaerobic bacteria and antibiotic therapy with cefotaxime, linezolid, and metronidazole was initiated. Drainage of the abscess 3 days later revealed purulent content. Cultures were positive for Streptococcus milleri, a facultative anaerobic gram-positive bacteria.

A thoracic CT demonstrated pulmonary arteriovenous fistulas up to $2 \mathrm{~cm}$ in diameter in middle lobe and lingula and the patient was diagnosed for Rendu-Osler-Weber syndrome (Fig. 3).

Neurological symptoms present at admission resolved. The patient was discharged awaiting embolization of vascular malformations.

\section{Discussion}

Rendu-Osler-Weber syndrome is a genetic, autosomal dominant disorder characterized by dilatation of small skin and mucosal blood vessels which leads to epistaxis and hemorrhage into the digestive tract. It affects 1 in 100,000 people.
The diagnostic criteria are recurrent epistaxis, multiple vascular dilatations, arteriovenous malformations or fistulas in the internal organs including lungs, liver, brain, or bone marrow, and a first-degree relative with the condition. ${ }^{3)} \mathrm{Pul}$ monary arteriovenous malformations predispose to the formation of brain abscess in immunocompetent patients due to hematogenous spread of pulmonary infectious foci. ${ }^{4}$

Brain abscess is a focal intracerebral infection that begins with a localized cerebritis and evolves to a collection of pus surrounded by a housing area; often caused by contiguous spread of infectious foci or hematogenous spread from a distant focus. It can occur at any age, more common in men than in women and the most common presenting symptoms are headache and altered mental status. Although there has been clear progress in diagnostic techniques and treatment in recent decades, brain abscesses still carry significant morbidity and mortality. Because of this, early diagnosis is crucial to optimize the use of antibiotics; the success of treatment is best when the etiologic agent is identified and antimicrobial therapy is targeted. ${ }^{5,6}$

$1 \mathrm{H}-\mathrm{MRS}$ instead of showing the signals of the protons as a grayscale image based on their relative signal strength shows the amounts as a spectrum, and metabolites in greater numbers have a greater peak height or greater area under the peak.

Typical 1H-MRS features of brain abscesses include high peaks of amino acids, lactate, alanine, acetate and succinate and, as the abscess cavity lacks normal brain tissue, there are no basic brain metabolites ( $\mathrm{N}$-acetylaspartate, creatine, and choline). Rupture of the cell membranes and proteolytic enzymes released by neutrophils produce cytosolic amino acids (valine, leucine, and isoleucine), bacterial fermentation leads to the production of lactate, acetate and succinate; and necrosis produces lactate and lipids. The presence of anaerobic bacteria tends to cause an increase of acetate and succinate while the absence thereof becomes more likely to be an infection by aerobic bacteria., 2,7-12)

Resonances of acetate with or without succinate on 1H-MRS favor an abscess of anaerobic bacterial origin and, in some cases, facultative anaerobic microbes. Nonvisibility 
of these resonances in anaerobic culture-positive brain abscesses could be explained by the relatively low concentration of these metabolites, below the sensitivity of the current scanner. ${ }^{13)}$ Considering a molecular method like polymerase chain reaction as gold standard for the categorization of bacteria in brain abscess, Bajpai et al. ${ }^{14)}$ found a sensitivity and specificity values of in vivo $1 \mathrm{H}-\mathrm{MRS}$ in culture positive samples of $90 \%$ and $100 \%$ for aerobic and $87.5 \%$ and $90 \%$ for anaerobic detection, respectively.

The evolution of brain abscesses can affect the 1H-MRS findings. Bacteria are present in the necrotic center and inflammatory cells are spread diffusely in the early cerebritis stage, while a larger necrotic center and many inflammatory cells characterize late cerebritis. Inflammatory cells decrease while fibroblasts increase in the early capsule stage, and few inflammatory cells are to be found in the late capsule stage. $1 \mathrm{H}-\mathrm{MRS}$ may exhibit chronological changes. Patients can show spectral changes on examinations before and after medical and surgical treatment, from the spectrum of a brain abscess (amino acids, acetate) to a single, nonspecific lactate peak. This spectral change may reflect the effectiveness of the treatment. ${ }^{15)}$ Further studies are needed to evaluate the correlation of $1 \mathrm{H}-\mathrm{MRS}$ with pathological staging. ${ }^{16)}$

\section{Conclusion}

It is possible to differentiate abscesses produced by anaerobic or aerobic bacteria based on the behavioral patterns of metabolites in 1H-MRS. The demonstration of amino acids suggests the diagnosis of brain abscess and the presence of acetate and succinate suggests anaerobic infection, this information is useful to facilitate diagnosis and therefore early treatment of these patients.

\section{Conflicts of Interest Disclosure}

None.

\section{References}

1) Möller-Hartmann W, Herminghaus S, Krings T, Marquardt G, Lanfermann H, Pilatus U, Zanella FE: Clinical application of proton magnetic resonance spectroscopy in the diagnosis of intracranial mass lesions. Neuroradiology 44: 371-381, 2002
2) Garg M, Gupta RK, Husain M, Chawla S, Chawla J, Kumar R, Rao SB, Misra MK, Prasad KN: Brain abscesses: etiologic categorization with in vivo proton MR spectroscopy. Radiology 230: 519-527, 2004

3) Nozaki T, Nosaka S, Miyazaki O, Makidono A, Yamamoto A, Niwa T, Tsutsumi Y, Aida N, Masaki H, Saida Y: Syndromes associated with vascular tumors and malformations: a pictorial review. Radiographics 33: 175-195, 2013

4) Al-Okaili RN, Krejza J, Wang S, Woo JH, Melhem ER: Advanced MR imaging techniques in the diagnosis of intraaxial brain tumors in adults. Radiographics 26(Suppl 1): S173-S189, 2006

5) Muzumdar D, Jhawar S, Goel A: Brain abscess: an overview. Int J Surg 9: 136-144, 2011

6) Prasad KN, Mishra AM, Gupta D, Husain N, Husain M, Gupta RK: Analysis of microbial etiology and mortality in patients with brain abscess. J Infect 53: 221-227, 2006

7) Rath TJ, Hughes M, Arabi M, Shah GV: Imaging of cerebritis, encephalitis, and brain abscess. Neuroimaging Clin N Am 22: 585-607, 2012

8) Lai PH, Ho JT, Chen WL, Hsu SS, Wang JS, Pan HB, Yang CF: Brain abscess and necrotic brain tumor: discrimination with proton MR spectroscopy and diffusion-weighted imaging. AJNR Am J Neuroradiol 23: 1369-1377, 2002

9) Dusak A, Hakyemez B, Kocaeli H, Bekar A: Magnetic resonance spectroscopy findings of pyogenic, tuberculous, and cryptococcus intracranial abscesses. Neurochem Res 37: 233-237, 2012

10) Chiang IC, Hsieh TJ, Chiu ML, Liu GC, Kuo YT, Lin WC: Distinction between pyogenic brain abscess and necrotic brain tumour using 3-tesla MR spectroscopy, diffusion and perfusion imaging. Br J Radiol 82: 813-820, 2009

11) Morais LT, Zanardi Vde A, Faria AV: Magnetic resonance spectroscopy in the diagnosis and etiological definition of brain bacterial abscesses. Arq Neuropsiquiatr 65: 1144-1148, 2007

12) Elshafey R, Hassanein O, Shakal A, Mokbel E: $1 \mathrm{H}$ proton MR spectroscopy and diffusion-weighted imaging in discrimination between pyogenic brain abscesses and necrotic brain tumors. The Egyptian Journal of Radiology and Nuclear Medicine 45: 889-896, 2014

13) Pal D, Bhattacharyya A, Husain M, Prasad KN, Pandey CM, Gupta RK: In vivo proton MR spectroscopy evaluation of pyogenic brain abscesses: a report of 194 cases. AJNR Am J Neuroradiol 31: 360-366, 2010

14) Bajpai A, Prasad KN, Mishra P, Gupta RK, Singh AK, Ojha BK: Multimodal approach for diagnosis of bacterial etiology in brain abscess. Magn Reson Imaging 32: 491-496, 2014

15) Dev R, Gupta RK, Poptani H, Roy R, Sharma S, Husain M: Role of in vivo proton magnetic resonance spectroscopy in the diagnosis and management of brain abscesses. Neurosurgery 42: 37-42; discussion 42-43, 1998

16) Akutsu H, Matsumura A, Isobe T, Anno I, Takano S, Itai $Y$, Nose $T$ : Chronological change of brain abscess in (1)H magnetic resonance spectroscopy. Neuroradiology 44: 574-578, 2002

Corresponding author:

Alex Roberto Ramírez Mejía, MD, Department of Diagnostic Radiology, Hospital Clínico San Carlos, Profesor Martín Lagos S/N 28040, Madrid 28040, Spain.

$\bowtie$ alex.ramirez.mej@gmail.com 